\title{
EL "AUTOMATIZADO BOX \& BLOCKS TEST" SISTEMA AUTOMÁTICO DE EVALUACIÓN DE DESTREZA MANUAL GRUESA
}

\author{
Edwin D. Oña ${ }^{1}$, Alberto Jardón ${ }^{1}$, Carlos Balaguer ${ }^{1}$, Alicia Cuesta ${ }^{2}$, María Carratalá ${ }^{2}$, Esther Monge ${ }^{2}$ \\ ${ }^{1}$ Robotics Lab, Universidad Carlos III de Madrid, Avda. Universidad 30, Leganés (Madrid), España \\ \{eona, ajardon, balaguer\}@ing.uc3m.es \\ ${ }^{2}$ Universidad Rey Juan Carlos, Avda. de Atenas s/n, Alcorcón (Madrid), España \\ \{alicia.cuesta, maria.carratala, esther.monge\}@urjc.es
}

\section{Resumen}

El cada vez más elevado número de personas que requieren atención médica para rehabilitación física o cognitiva (post-traumática, post-operatoria o por envejecimiento), supone cada vez mayor saturación en los servicios médicos. Esto repercute de forma negativa en la calidad del tratamiento impartido. El proyecto RoboHealth, intenta contribuir a paliar dicha situación, proporcionando asistencia al personal sanitario en procesos de rehabilitación física. RoboHealth abarca dos aspectos durante el proceso de rehabilitación motora: la evaluación y la intervención. En este artículo, se presenta el diseño de un sistema automatizado de evaluación de destreza manual gruesa basado en el test Box and Blocks. A través de cámaras $R G B$ y nube de puntos, se monitoriza la ejecución del test, obteniendo de forma automática el total de cubos desplazados por el usuario, además de datos adicionales como el tiempo empleado en el desplazamiento entre cada cubo y su color. La información se puede visualizar a través de una interfaz gráfica que además, ordena y registra de forma automática los resultados de cada sesión para cada usuario. En primer lugar, se muestra el desarrollo del algoritmo de conteo, la implementación de la interfaz de visualización y registro de datos, así como los resultados de una prueba piloto. Finalmente, se presentan las conclusiones y futuros trabajos.

Palabras clave: Rehabilitación, Evaluación, Automático, Extremidad superior, Destreza manual

\section{INTRODUCCIÓN}

En la actualidad, la presencia de sistemas de asistencia robóticos durante procesos de rehabilitación física es cada vez mayor. Adicionalmente, esto se puede ver reflejado con el desarrollo de sistemas comerciales para la rehabilitación basados en órtesis activas, comúnmente denominados exoesqueletos. Es el caso de sistemas utilizados para terapia de miembro superior como los desarrollados por Tyromotion [13] (DIEGO ${ }^{\circledR}$, AMADEO $^{\circledR}$, PABLO $\left.{ }^{\circledR}, \mathrm{MYRO}^{\circledR}\right)$. Toda su gama, cuenta con el software TyroS el cual permite a los sistemas antes mencionados, interactuar con el paciente (motivación), evaluar la ejecución de la tarea, y registrar los resultados. Otro fabricante consolidado es Hocoma [2] que comercializa, entre otros, dos sistemas basados en el concepto de terapia para miembro superior como el VALEDO` ${ }^{\circledR}$, o el sistema ARMEO ${ }^{\circ}$. Para rehabilitación de miembro inferior desarrollaron LokoMat`, sistema de rehabilitación física para el entrenamiento de la función de la marcha. El tratamiento es potenciado además por una interfaz de usuario basada en realidad virtual, que intenta motivar al paciente; puede ser utilizado por adultos y niños. Estos sistemas, si bien cuentan con sus propios métodos de registro de datos y evaluación de la ejecución de la tarea, son también plataformas cerradas, costosas, poco flexibles y enfocadas más a la rehabilitación en sí misma.

Debido a que la rehabilitación es un proceso laborioso y de costosa intervención, evaluar su efectividad terapéutica es particularmente importante [14]. Esta valoración es comúnmente realizada por los propios profesionales sanitarios. Utilizan escalas estandarizadas para intentar conservar la objetividad en la evaluación, pero en la práctica están sujetas a la subjetividad del observador. En algunos casos, los métodos de evaluación están compuestos por ejercicios bien definidos y basados en escalas numéricas, que podrían ser susceptibles de ser automatizados. Así, se facilitaría una evaluación objetiva de la condición física de los sujetos a tratar. Además, se provee al rehabilitador de más tiempo para valorar los resultados, y en base a ellos, reconducir el método de terapia aplicado, modificar su nivel de dificultad, o finalizar el proceso.

Para automatizar un proceso de rehabilitación, 
se deben valorar: el método, las métricas a extraer, así como el grado de aceptación tanto por los usuarios como por el personal sanitario. En el diseño de sistemas de asistencia a la rehabilitación, si bien el enfoque se centra en el sujeto a tratar, es importante sistematizar la captura y comprensión de los requisitos que demandan los terapeutas para facilitar una integración más sencilla de la tecnología en sus actividades diarias [11]. Respecto al método, resultan más susceptibles de ser automatizadas aquellas pruebas que se administran sin contacto directo del profesional. Para el caso de las métricas, se debe valorar cuales dan información relevante y cuales son las menos invasivas posibles para el sujeto evaluado.

\subsection{ESCALAS DE EVALUACIÓN TRADICIONALES}

La medición de los resultados de la asistencia sanitaria, es un componente esencial para determinar la eficacia de una terapia y, por lo tanto, la prestación de asistencia médica basada en la evidencia [14]. Los primeros medidores genéricos de nivel de discapacidad fueron el Barthel Index (BI), desarrollado en los años 50' planteado como un índice simple de independencia que consta de 10 items, y por otro lado, el Functional Independence Measure (FMI) desarrollado en los años 80' como un instrumento para obtener una medición más completa de discapacidad que consta de 18 items repartidos en seis categorías. En 2001, la Organización Mundial de la Salud (OMS), adoptó la Clasificación Internacional de Funcionalidad, Discapacidad y Salud, comúnmente conocida como la CIF [17], que proporciona un marco común para describir las consecuencias de las condiciones de salud y, un estándar internacional para describir y medir la salud y la discapacidad. En el ámbito clínico, la CIF se utiliza para la evaluación del estado funcional, la fijación de objetivos, planificación y el seguimiento del tratamiento, así como la medición de resultados.

A día de hoy, se pueden encontrar múltiples escalas de valoración utilizadas según la sintomatología. En rehabilitación post accidente cerebro vascular (ACV), por ejemplo, las escalas se pueden clasificar según estructura del cuerpo, actividad y participación. Entre las escalas habitualmente utilizadas en la categoría "Estructura del cuerpo" se encuentran el Fugl-Meyer Assessment (FMA) o el Modified Ashworth Scale (MAS); en la categoría "Actividad" se utilizan, por ejemplo, el Barthel Index (BI), el Action Research Arm Test (ARAT), el Box and Blocks Test (BBT), el Wolf Motor Function Test (WMFT) o el Timed Up $\&$ Go Test (TUG); y en la categoría de "Participación" por ejemplo el Stroke Impact Scale (SIS) [12].

\subsubsection{El Box \& Blocks Test}

El BBT es un sistema de medida individual de la destreza y coordinación manual, clínicamente validado. El test está formado por una caja de madera con dos compartimientos cuadrados de $290 \mathrm{~mm}$ de lado, y 150 cubos de madera de $25 \mathrm{~mm}$. Entre los dos compartimientos se ubica una barrera de 100 $\mathrm{mm}$ de alto, que el usuario debe sobrepasar con su mano para que el conteo del cubo sea válido. En la Figura 1, se muestra la estructura de la caja usada para el test.

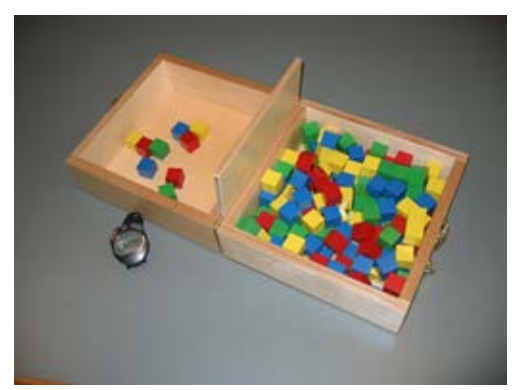

Figura 1: Box \& Blocks Test y cronómetro manual

El objetivo del test es desplazar la mayor cantidad posible de cubos de un lado de la caja al otro en un minuto. Para la puntuación, el terapeuta cuenta manualmente el total de cubos desplazados. El desarrollo del test se comprende de tres etapas: un periodo de entrenamiento de 15 segundos, ejecución del test con la mano dominante o no afectada durante un minuto, y finalmente ejecución del test con la mano no dominante o afectada durante un minuto. Para que el desplazamiento del cubo sea válido, la mano del individuo debe sobrepasar la barrera central de la caja. No se cuentan los cubos que sean lanzados desde una parte de la caja a la otra. Además, el desplazamiento de cubos se debe realizar de uno en uno, y en caso de desplazar más de un cubo a la vez, se contarán como uno solo. Para realizar el test, se ubica el BBT sobre una mesa y el usuario desde una posición sentada delante del test, realiza el ejercicio. El evaluador, lee las instrucciones del test al individuo antes de iniciar la evaluación. Las reglas completas, así como las instrucciones que se le deben impartir a los usuarios del test, se recogen en [7],[10].

\subsection{MÉTODOS AUTOMATIZADOS DE EVALUACIÓN}

Actualmente, además de proyectos enfocados al desarrollo de ayudas para rehabilitación y de los sistemas comerciales antes mencionados, se pueden encontrar proyectos de investigación enfocados en automatizar métodos de evaluación basados en escalas neuromotoras habitualmente uti- 
lizadas en centros asistenciales, o que plantean implementar sistemas con escalas nuevas que se comparan con las escalas tradicionales. Ubicados en la categoría de estructura del cuerpo, se encuentran varios trabajos. Por ejemplo, en [8] se propone un método de evaluación basado en sensores de bajo coste que registran datos de los movimientos del usuario. Sus resultados se han comparado con los obtenidos utilizando el habitual FMA obteniendo resultados similares. Afirman que pueden automatizar la mayoría del FMA. En [16] se presenta otro intento de automatizar parte del FMA, en este caso, utilizando acelerómetros y centrándose en puntuaciones para movimientos de hombro y codo. Uno de los síntomas más complejos después de sufrir un ACV es el déficit visuoespacial. Para su diagnóstico y evaluación se utilizan una serie de pruebas basadas en "lápiz y papel" (que implican tareas como el tachado de un objetivo, bisección de una línea o copiar una forma geométrica). En [6] se propone un modelo basado en el análisis de los dibujos, y los resultados se comparan con los obtenidos con el Rivermead Behavioural Inattention Test (BIT). Los dibujos se realizan en un folio de papel superpuesto sobre una tablet. Los trazos digitalizados, son procesados por ordenador, se extraen sus características y se analizan los resultados para dar la puntuación.

También, se encuentran trabajos que buscan automatizar escalas dentro de la categoría de actividad. En [9] se propone un sistema nuevo llamado Rejoyce Arm and Hand Function Test (RAHFT), enfocado a la evaluación de destreza manual. Utiliza la estación de trabajo Rejoyce en la que los participantes realizan tareas de destreza manual bajo la apariencia de juegos de ordenador. Los autores afirman que es la primera prueba de destreza manual que no depende del juicio humano, ofreciendo una evaluación de los resultados cuantitativa, estandarizada y que se puede administrar de forma remota. En [4] se implementa un sistema automático que evalúa de manera objetiva la funcionalidad del miembro superior en rehabilitación post ACV. El sistema realiza un post-procesado de datos experimentales, obtenidos durante la realización de tareas de alcance utilizando el sistema robótico KINARM. En este caso, los resultados son comparados con el Chedoke-McMaster Stroke Assessment Scale (CMSA). Una evaluación automática basada en el WMFT se plantea en [15]. Utilizando sensores que los usuarios deben llevar puestos, se estima el tiempo que tardan en completar 7 de las 17 tareas del test. Otro trabajo en curso se presenta en [5], donde se busca automatizar el ARAT. En dicho trabajo, se ha planteado la automatización del sub-test 4 del ARAT, por medio de la sensorización de uno de los objetos utilizados en la tarea, en este caso un cubo de 7.5 cm. Finalmente, en [3] se presenta el Digital Box and Blocks Test (DBBT), con el objetivo de automatizar el test tradicional con el mismo nombre. En este trabajo, utilizando un sensor Kinect ${ }^{\circledR}$, se implementa un algoritmo para contar los cubos desplazados con un índice de acierto del 100\% hasta 20 cubos. Además, detecta la mano y sus movimientos.

De los sistemas revisados se puede observar que hay varios trabajos enfocados en detectar los movimientos de miembro superior, ya sea por medio de sensores portables que lleve puesto el sujeto, senzorizando los objetos usados en el test, o por sistemas de visión por ordenador. Además, un objetivo común es obtener plataformas de evaluación automática que sean objetivas, que tengan repetividad, capacidad de diagnóstico y que sean dinámicas, pudiendo aportar información adicional a la que se obtendría con la escala tradicional.

\section{METODOLOGÍA}

Por su amplia utilización en entornos clínicos como sistema de evaluación en procesos de rehabilitación de personas que han sufrido un $\mathrm{ACV}$, y además, por sus características adecuadas para la automatización, se plantea el estudio del BBT. La configuración del sistema propuesto se muestra en la Figura 2, y consta de una estructura ligera y portable en forma de cubo, que se ubica sobre una mesa estándar. En la parte superior de la estructura, se coloca un sensor Kinect ${ }^{\circledR}$ para Windows ${ }^{\circledR}$, mediante el cual se detecta el número de cubos desplazados, así como los movimientos de la mano del individuo durante el ejercicio. En el centro de la estructura y sobre la mesa se sitúa el la caja tradicional del BBT.

El sistema propuesto busca cubrir los siguientes objetivos:

a) Automatización del test: partiendo del conteo automático de cubos, y obteniendo información adicional de los movimientos del usuario.

b) Interfaz de visualización: le sirve al terapeuta para supervisar la ejecución del test en el lugar in situ o a distancia, y guía al usuario durante el test.

c) Registro automático de datos: los resultados del test se almacenan en una base de datos con la que se puede generar un histórico de las sesiones previas. Además, se pueden grabar vídeos de las sesiones. 


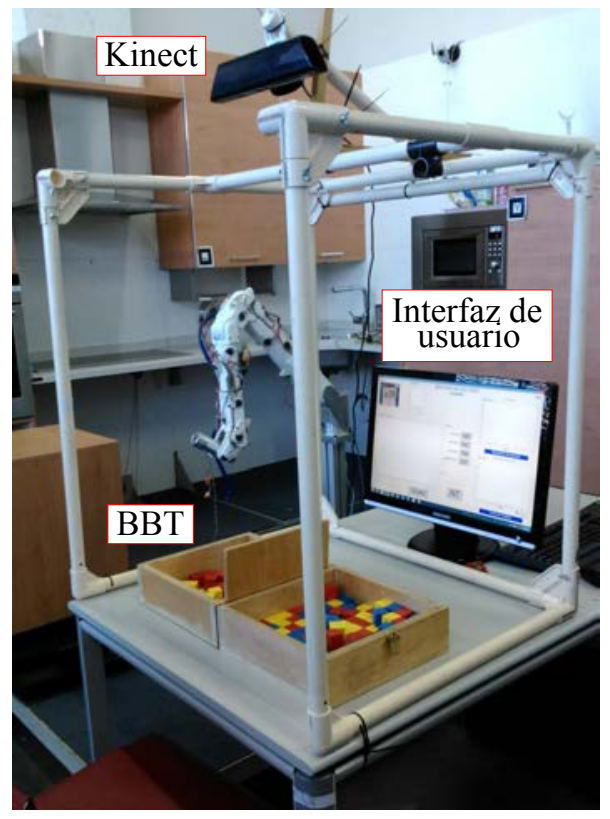

Figura 2: Estructura propuesta para automatización del BBT

De esta manera, el sistema propuesto denominado el Automatizado Box \& Blocks Test (ABBT), va un paso más allá que el sistema tradicional y el DBBT [3]. ya que no solo se implementa un algoritmo para obtener una puntuación automática, sino que se aborda también la administración automática del test por medio de la interfaz gráfica.

\section{$2.1 \quad$ AUTOMATIZACIÓN DEL TEST}

El ABBT, se implementa bajo Windows 10 utilizando a nivel software Matlab ${ }^{\circledR}$ R2015b, que incluye paquetes de soporte hardware para dispositivos como la Kinect ${ }^{\circledR}$ para Windows ${ }^{\circledR}$ v1. Además, Matlab ${ }^{\circledR}$ cuenta con potentes herramientas y librerías para procesado de imágenes, ofreciendo también el entorno de GUIDE para el desarrollo de interfaces gráficas. El proceso de conteo automático de cubos se desarrolla en tres etapas: a) Detección del borde de las cajas; b) Segmentación de las imágenes por color; y c) Validez del intento.

\subsubsection{Detección del borde}

El sensor Kinect ${ }^{\circledR}$ se ubica en la parte superior de la estructura (ver Figura 2), y a una distancia aproximada de $1 \mathrm{~m}$ respecto de la mesa. Se fija su posición a través de un soporte de plástico ABS fabricado con una impresora de prototipado rápido. Ya que la posición de la Kinect ${ }^{\circledR}$ en la estructura se mantiene invariable, conseguimos que la distancia del sensor al borde de la caja del BBT también se mantenga constante.

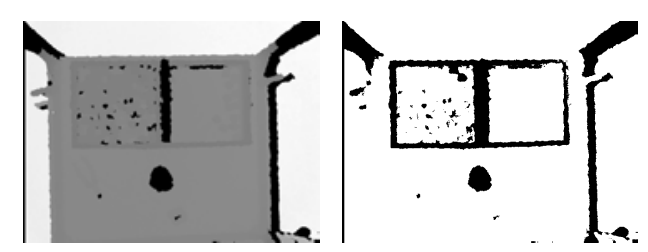

(a)

(b)

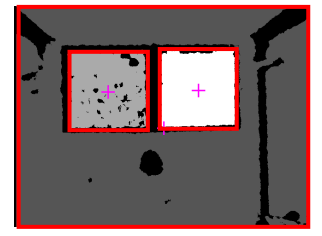

(c)

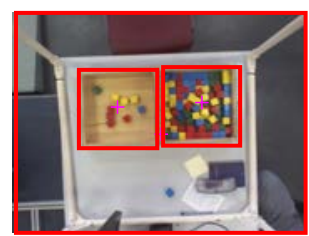

(d)
Figura 3: Secuencia para detección del borde del BBT: a) Nube de puntos, b) Umbral por altura, c) Detección de los compartimientos y, d) Regiones de interés sobre imagen RGB

En la Figura 3, se muestra el proceso para detectar el borde de la caja, y diferenciar los dos compartimientos. En primer lugar, se captura la nube de puntos (Figura 3-a) y se le aplica un umbral de profundidad, en este caso $90 \mathrm{~cm}$. Los puntos por debajo de esta altura se descartan, incluyendo la mesa (Figura 3-b). Con la imagen umbralizada, se aplican operaciones morfológicas para eliminar ruido y etiquetar las zonas detectadas. Con la imagen mejorada, se puede extraer las características basadas en varios algoritmos. En este caso, utilizamos la función 'BoundingBox' para obtener los rectángulos en la imagen, así como su centroide. Basándonos en las coordenadas de los centroides, se puede diferenciar entre el compartimiento de la izquierda y el de la derecha (Figura 3-c). Además, añadimos un filtro adicional por área ya que el área de los compartimientos es conocida. El identificar los compartimientos es importante de cara a generar una máscara para la segmentación por color en el conteo de los cubos. Finalmente, se pueden superponer las regiones de interés sobre la imagen en color de la escena (Figura 3-d).

\subsubsection{Segmentación por color}

Teniendo en cuenta el gran contraste entre los colores de los cubos del BBT (rojo, azul, amarillo y verde), y también respecto del fondo de la caja de color haya, se opta por segmentar y procesar las imágenes capturadas en color RGB, para detectar y contar los cubos. Tras capturar la imagen en color con una resolución de 640×480 píxeles, se procesa la imagen para buscar las zonas de cada color por separado. Primero, se convierte la imagen en color a blanco y negro, sintonizando cada color según umbrales diferentes. Dichos 
parámetros se ajustan de forma automática tras la calibración de la luz (intensidad de la escena), para lo cual se depositan 3 cubos de cada color en uno de los compartimientos de la caja. Sobre la imagen umbralizada, se aplica un enmascaramiento para procesar únicamente la región de interés. Dicha región se corresponde con el lado vacío de la caja, y que depende de si el test se realiza para la mano afectada o no afectada del sujeto. A continuación, se aplica una serie de operaciones morfológicas (apertura, cierre, erosión y etiquetado), repitiendo la misma secuencia para cada color por separado. Finalmente, se hace un primer conteo de los cubos detectados, según el color, en la imagen capturada.

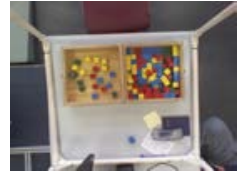

(a)

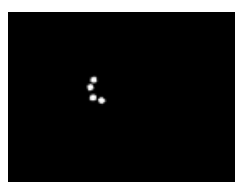

(d)

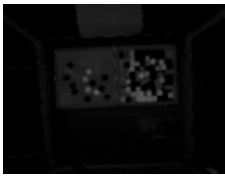

(b)

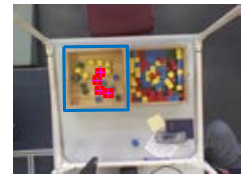

(e)

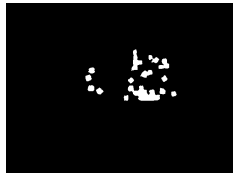

(c)

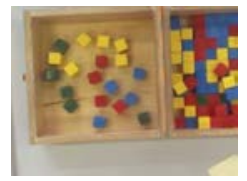

(f)
Figura 4: Procesado de imagen para color rojo: a) Imagen en color, b) Umbralización a escala de grises, c) Conversión a blanco y negro, d) Enmascaramiento de región de interés, e) Cubos detectados sobre imagen RGB, f) Zoom de región de interés

En la Figura 4, se muestra las partes principales del procesado de imagen para la detección de cubos de color rojo. Se aprecia la captura de imagen (Figura 4-a), la conversión de la imagen en color a escala de grises utilizando un umbral (Figura 4-b) y después la conversión a blanco y negro (Figura 4-c). Se aplica la máscara generada a partir de los bordes de la caja para seleccionar la zona de interés (Figura 4-d) y finalmente, se enmarcan las zonas de color rojo correspondientes a los cubos detectados, en este caso cuatro (Figura 4-e). En la Figura 4-f se muestra un zoom de la imagen en color. Para detectar el resto de cubos, el proceso es análogo utilizando la misma máscara y los umbrales correspondientes.

\subsubsection{Validación del intento}

Como es de esperar, es posible que algunos individuos presenten problemas para coger un solo cubo, pudiendo coger dos a las vez, debido a la falta de sensibilidad y a la elevada espasticidad en sus manos. En estos casos, y según la normativa del test [7] se deben descontar los cubos adicionales y contar como uno solo. Para solventar esta casuística en la ejecución del ABBT, se utiliza el vector de tiempo para comparar eventos muy cercanos. Partiendo de que un individuo sano tarda alrededor de un segundo en desplazar un cubo, durante el procesado instantáneo de la imagen se detecta si han aparecido más de un cubo en tiempos cercanos y menores de un segundo. En este caso, se descartan los eventos adicionales y solo se suma un cubo al contador global.

\subsection{INTERFAZ GRÁFICA}

Utilizando el entorno de desarrollo de aplicaciones de usuario GUIDE de Matlab ${ }^{\circledR}$, se está implementando una interfaz gráfica en la que se muestra el desarrollo del test. En este caso, la interfaz está orientada al terapeuta. En ella, además de visualizar la detección de los cubos, se presentan opciones de selección del perfil del usuario, que carga de forma automática la información del sujeto almacenada en una base de datos local. Entre la información almacenada, tenemos el dato de cuál es la mano afectada, y de acuerdo a esto, el ABBT selecciona la máscara adecuada (región de la caja).

Por otro lado, además de la cuenta de los cubos según el color, se muestran dos ventanas con gráficos. En la primera, se presentan los resultados de cubos y tiempos obtenidos en la primera etapa (mano no afectada). En la segunda ventana, y tras la segunda etapa del test (mano afectada) se presenta un gráfico comparativo de la sesión actual junto con las sesiones previas. En la Figura 5 se muestra la interfaz gráfica implementada.

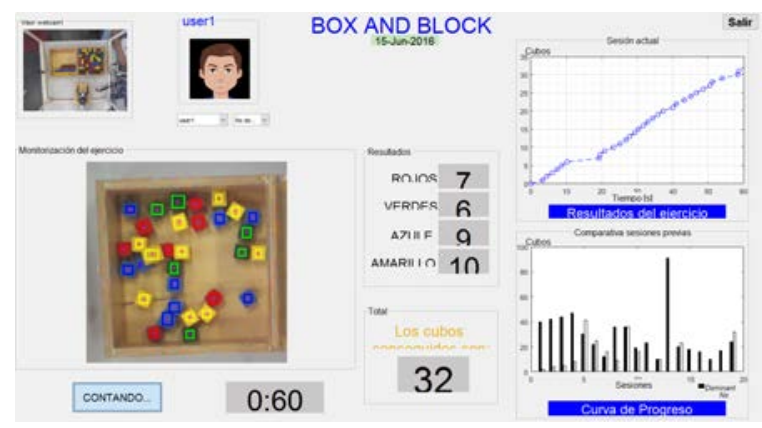

Figura 5: Interfaz gráfica para el ABBT

\subsection{REGISTRO AUTOMÁTICO DE RESULTADOS}

Un aspecto importante en la actualidad, es el procesamiento y almacenamiento digital de datos. Esta tendencia, apoyada por la introducción de las tecnologías de la información y comunicación (TIC), está cada vez más presente en centros asis- 
tenciales para la gestión digital de los datos de los pacientes. Este planteamiento, junto con el concepto de telemedicina, son firmes propuestas de futuro [1]. En esta línea, la interfaz del ABBT es capaz de almacenar de forma automática los resultados obtenidos en la ejecución del test. Guarda en una base de datos local, organizados según el usuario y número de sesión, los valores de número total de cubos desplazados para el caso de mano afectada y no afectada, así como el tiempo de desplazamiento entre los cubos. La organización por número de sesión, permite visualizar de forma instantánea un informe histórico de los datos, pudiendo fácilmente ver la progresión y eficacia de la terapia aplicada, y en caso necesario reforzarla o reconducirla.

\section{RESULTADOS}

La efectividad del algoritmo de conteo de cubos, se mide en laboratorio en base a reiteradas pruebas con la ayuda del personal técnico realizando el test. Se obtiene, que el sistema presenta una efectividad en el conteo de cubos del 100\% hasta un número de 25 cubos.

Para tener una primera impresión del sistema en una situación real, se ha realizado una prueba piloto con un usuario de rehabilitación. Se planteó que el sujeto realizase el test completo de la misma forma en que se llevaría a cabo en un entorno hospitalario, pero con la mínima intervención de profesionales sanitarios. La prueba tuvo lugar en las instalaciones del Laboratorio de Robótica Asistencial en el Parque Científico y Tecnológico de la Universidad Carlos III de Madrid (UC3M). La configuración del sistema, ha sido la mostrada en anterior Figura 2. Para ejecutar el software, se empleó un ordenador portátil Intel ${ }^{\circledR}$ Core i7 junto con una cámara Kinect ${ }^{\circledR}$ para Windows ${ }^{\circledR}$ v1. El algoritmo implementado es capaz de procesar 2.9 imágenes por segundo (344 ms de ciclo).

\subsection{PARTICIPANTES}

Para este ensayo piloto se contó con la participación de una persona, mujer de 55 años, con ACV hemorrágico de la arteria cerebral-media izquierda, que cumplió con los siguientes criterios de selección: a) Afectación de la extremidad superior; b) Capacidad de agarre; c) Espasticidad según la escala de Ashworth Modificada inferior a 2 ; d) Capacidad para comprender las instrucciones (Mini-mental test superior a 24 puntos). Además, se contó con la participación de dos terapeutas que supervisaron el correcto desarrollo del test en todo momento.

\subsection{ENSAYO PILOTO}

Se buscó el análisis de la eficacia del sistema automático, en una sola sesión, en un sujeto con patología neurológica que presentaba afectación de la extremidad superior. Se propuso al sujeto utilizar el ABBT por sí solo, sin ayuda del equipo de trabajo. Para guiar al usuario durante la ejecución, el ABBT reproduce instrucciones por medio de un sintetizador de voz. Dichas instrucciones, son similares a las impartidas por un profesional sanitario con el tradicional BBT. Como indican las reglas del test, tras el periodo de prueba, el individuo procedió a realizar el test empezando con su mano dominante. A continuación, realiza el test con la mano afectada. Al finalizar cada fase, uno de los profesionales sanitarios presentes, procede a contar los cubos desplazados para comparar los resultados con los obtenidos por el sistema automatizado. El sujeto lleva algún tiempo en el tratamiento de rehabilitación y se encontraba familiarizado con el test tradicional. Por lo tanto, pudo aportar una valiosa comparación entre el BBT y el ABBT.

\subsection{RESULTADOS DEL ENSAYO}

En la Figura 6, se muestran los resultados de cubos obtenidos y el instante de tiempo en el que han sido detectados, para el caso de la mano no afectada (32 cubos) y afectada (3 cubos). Los resultados conseguidos con el ABBT, han sido desiguales obteniendo un porcentaje de acierto en el conteo automático de cubos del $100 \%$ para la mano afectada y un $74 \%$ para la mano no afectada.

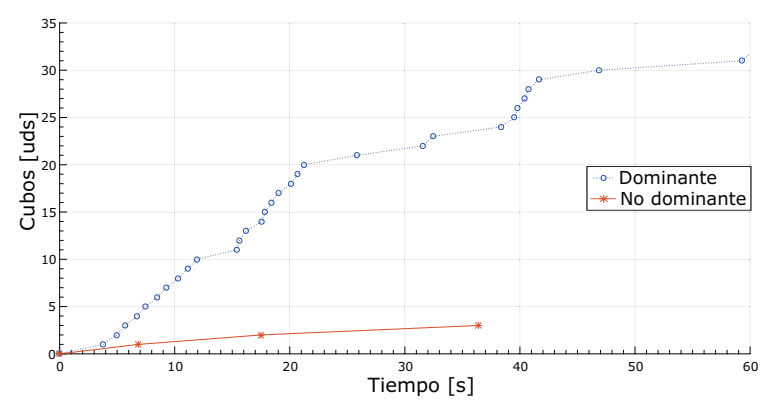

Figura 6: Resultados detección de cubos con el ABBT

Cabe destacar que, siguiendo las instrucciones trasmitidas por los mensajes de voz, el sujeto fue capaz de completar satisfactoriamente todo el ABBT a través de la interfaz implementada, lo que equivale al desarrollo completo del BBT. De esta manera, se comprueba la viabilidad para administrar el ABBT de forma automática, y plantea la posibilidad de realizar un ensayo con un mayor número de personas. 


\section{CONCLUSIONES}

En este artículo, se presentó el desarrollo hardware y software para automatizar el test de destreza y coordinación manual BBT. A través de un ensayo piloto se ha realizado una primera valoración de la viabilidad del ABBT para ser administrado de forma automática, con mínima participación del terapeuta. También, para medir la efectividad del sistema propuesto en una situación con un usuario real. Como resultado, el sistema automático es altamente valorado por el usuario, quien pudo seguir su ejecución sin mayor dificultad a través de la interfaz implementada. Esto refuerza la validez dada por parte del personal sanitario.

Se ha comprobado que el sistema dispone de una sensorización adecuada, y que ésta provee más información que el método tradicional. Dicha información, está almacenada directamente en el registro del paciente, facilitando la disponibilidad de un historial clínico actualizado. En este caso, se ha conseguido detectar el color de los cubos obtenidos, así como el instante de tiempo en el que han sido desplazados, presentando al final de la sesión un informe histórico comparativo de las sesiones previas. Respecto de trabajos similares como en DBBT [3], no solo se ha desarrollado un algoritmo para el conteo de cubos, sino que el ABBT implementa una interfaz gráfica con la que se consigue completar satisfactoriamente todas las fases del BBT (entrenamiento, mano no afectada y mano afectada), abordando la administración automática del test. Además, la interfaz permite visualizar el desarrollo de la prueba, así como registrar el vídeo y los datos de la sesión para un análisis posterior si se requiere. El análisis de esta información temporal, genera información adicional que puede sugerir al terapeuta problemas durante el desarrollo de la prueba, como una reacción a fatiga, mejorando más el DBBT. En relación con el algoritmo de conteo, el ABBT discrimina si el usuario coge dos o más cubos, no contabilizando los cubos extra en el resultado final. Respecto a la precisión en el conteo, el ABBT presenta un índice de acierto del $100 \%$ para 25 cubos, respecto de los 20 del DBBT. Durante el ensayo piloto, se ha podido apreciar que la efectividad del algoritmo de conteo de cubos al cambiar de entorno, se ha visto disminuida con respecto a la obtenida en laboratorio. Esta disminución es atribuida, por una lado al no conteo de cubos en la mano no afectada debido a la mayor velocidad de desplazamiento, y por otro lado, al cambio de condiciones de luz ambiental que introducen falsos positivos durante la realización del ensayo. El proceso de calibración debe ser mejorado en desarrollos futuros.
Finalmente, el uso de sistemas automatizados permite mejorar también, de cara al usuario, el proceso de evaluación, ya que se pueden incluir sistemas que además de registrar los datos de la evaluación, permitan motivar al usuario haciendo el proceso más amigable e incluso divertido. Así, otra línea futura sería incluir una interfaz de usuario basada en serious games con la que introducir un grado de motivación e incluso competitividad en el proceso de evaluación.

\section{Agradecimientos}

La investigación que lleva a estos resultados ha recibido financiación del proyecto ROBOHEALTH-A (DPI2013-47944-C4-1-R), financiado por el Ministerio de Economía y Competitividad español y del proyecto RoboCity2030III-CM (S2013 / MIT-2748) , financiado por Programas de Actividades de I + D en la Comunidad de Madrid y co-financiado por los Fondos Estructurales de la UE.

\section{Referencias}

[1] European Commission EC, (2012) eHealth Action Plan 2012-2020 - Innovative healthcare for the 21st century.

[2] Hocoma, https://www.hocoma.com/, [Web; accedido el 02-04-2016]

[3] Hsiao, C. P., et al., (2013). The Digital Box and Block Test Automating traditional poststroke rehabilitation assessment. Pervasive Computing and Communications Workshops (PERCOM Workshops), pp. 360-363.

[4] Jung, J. Y., et al., (2008) A hierarchical ensemble model for automated assessment of stroke impairment.IEEE International Joint Conference on Neural Networks IJCNN, pp. 3187-3191.

[5] Lee, T. K., et al., (2015). Automated scoring of rehabilitative tests with singular spectrum analysis. Signal Processing Conference (EUSIPCO), pp. 2571-2575.

[6] Liang, Y., et al.,(2010). A learning model for the automated assessment of hand-drawn images for visuo-spatial neglect rehabilitation. Neural Systems and Rehabilitation Engineering, 18(5), pp 560-570.

[7] Mathiowetz, V., et al., (1985) Adult Norms for the Box and Block Test of Manual Dexterity, Am J Occup Ther, pp 386 - 391.

[8] Otten, P., et al.,(2015). A Framework to Automate Assessment of Upper-Limb Motor 
Function Impairment: A Feasibility Study. Sensors, 15(8),pp 20097-20114.

[9] Prochazka, A., et al.,(2015). A fully automated, quantitative test of upper limb function. Journal of motor behavior, 47(1), 19-28.

[10] Rehabilitation Measures Database, http:// www.rehabmeasures.org/, [Web; accedido el 24-02-2016]

[11] Saborowski, M., et al., (2015) "How do you care for technology?" - Care professionals' experiences with assistive technology in care of the elderly, Technological Forecasting and Social Change, pp 133 - 140.

[12] Salter, K., et al, (2013) Chapter 21: Outcome Measures in Stroke Rehabilitation, Handbook of clinical neurology.

[13] Tyromotion Inc, http://tyromotion.com/, [Web; accedido el 22-05-2016]

[14] Van der Putten, J. J. M. F., et. al. (1999). Measuring change in disability after inpatient rehabilitation: comparison of the responsiveness of the Barthel Index and the Functional Independence Measure. Journal of Neurology, Neurosurgery $\& 3$ Psychiatry, 66(4), pp 480484.

[15] Wade, E., et al., (2010). Automated administration of the wolf motor function test for post-stroke assessment. Pervasive Computing Technologies for Healthcare (PervasiveHealth), pp. 1-7.

[16] Wang, J., et al., (2014). Automated fuglmeyer assessment using SVR model. Bioelectronics and Bioinformatics (ISBB), pp. 1-4.

[17] World Health Organization WHO, (2001)International Classification of Functioning, Disability and Health (ICF). 